\title{
A system for automatic recording and analysis of motor activity in rats
}

\author{
Francisco J. Heredia-López • Rossana M. May-Tuyub • \\ José L. Bata-García • José L. Góngora-Alfaro • \\ Fernando J. Álvarez-Cervera
}

Published online: 16 June 2012

(C) Psychonomic Society, Inc. 2012

\begin{abstract}
We describe the design and evaluation of an electronic system for the automatic recording of motor activity in rats. The device continually locates the position of a rat inside a transparent acrylic cube $(50 \mathrm{~cm} / \mathrm{side})$ with infrared sensors arranged on its walls so as to correspond to the $x$-, $y$-, and $z$-axes. The system is governed by two microcontrollers. The raw data are saved in a text file within a secure digital memory card, and offline analyses are performed with a library of programs that automatically compute several parameters based on the sequence of coordinates and the time of occurrence of each movement. Four analyses can be made at specified time intervals: traveled distance $(\mathrm{cm})$, movement speed $(\mathrm{cm} / \mathrm{s})$, time spent in vertical exploration (s), and thigmotaxis (\%). In addition, three analyses are made for the total duration of the experiment: time spent at each $x-y$ coordinate pair ( $\mathrm{min})$, time spent on vertical exploration at each $x-y$ coordinate pair (s), and frequency distribution of vertical exploration episodes of distinct durations. User profiles of frequently analyzed parameters may be created and saved for future experimental analyses, thus obtaining a full set of analyses for a group of rats in a short time. The performance of the developed system was assessed by recording the spontaneous motor activity of six rats, while their behaviors were simultaneously videotaped for manual analysis by two trained observers. A high and significant correlation was found between the values measured by the electronic system and by the observers.
\end{abstract}

F. J. Heredia-López • R. M. May-Tuyub • J. L. Bata-García •

J. L. Góngora-Alfaro $\cdot$ F. J. Álvarez-Cervera $(\triangle)$

Departamento de Neurociencias, Centro de Investigaciones

Regionales "Dr. Hideyo Noguchi”,

Universidad Autónoma de Yucatán,

Mérida, Mexico

e-mail: acervera@uady.mx
Keywords Motor activity · RISC microcontroller · Infrared sensor $\cdot$ SD card $\cdot$ Automatic system $\cdot$ Caffeine

A variety of techniques are used to evaluate an array of motor activity alterations in live animals, such as rats. For instance, some methods are based on infrared light systems to detect motor activity (Clarke \& Smith, 1985; Czech, 1984; Geyer, Russo, \& Masten, 1986). Another tactic is the use of systems based on video cameras (Bailoo, Bohlen, \& Wahlsten, 2010; Bonatz, Steiner, \& Huston, 1987; Dielenberg, Halasz, \& Day, 2006; Golani, Benjamini, \& Eilam, 1993; Hopper, Kernan, \& Wright, 1990; Noldus, Spink, \& Tegelenbosch, 2001; Pan, Lee, \& Lim, 1996; Sams-Dodd, 1995; Schwarting, Goldenberg, Steiner, Fornaguera, \& Huston, 1993). Vittorio, Scannapieco, and Renzi (2006) presented a Doppler radar-based system. The systems based on infrared motion detectors (Kramer, 1998) or infrared pyroelectric detectors that measure the radiated body heat of the animal (Masuo, Matsumoto, Morita, \& Noguchi, 1997) are inexpensive, but they can only measure global activity patterns (e.g., circadian rhythms), without quantifying the path trajectory or specific motor behaviors. Another system allowed for calculating the position and trajectory of a rat by measuring its center of gravity while walking in a circular open field standing on three strain gauge transducers placed in a triangular array in the periphery of the floor (Gapenne, Simon, \& Lannou, 1990). In all of these systems, a dedicated personal computer (PC) is needed to control the data-collecting device.

Brockwell, Ferguson, and Beninger (1996) described a photo-sensor-based system for monitoring place conditioning and motor activity in rats within conditioning boxes. Each of these boxes used a small embedded computer (microprocessor, RAM, ports, timer, and digital input- 
output lines). Such a system allowed researchers to run several experiments simultaneously. A PC (Macintosh Plus) was used to create a network in which it was the "master" that sent experimental conditions to each embedded computer (acting as a "slave"), and at the end of the experiments it downloaded the collected data from every one of them. However, the PC was not necessary during the actual experiments.

Another activity system is offered by Columbus Instruments, which is infrared photo-cell-based and capable of separating ambulatory from total activity to provide an indication of grooming, scratching, and nonambulatory activities (Columbus Instruments, 2011). With the addition of an optional photocell sensor array located above the subject, it provides vertical activity monitoring as well. The system also provides analog voltage outputs that are associated with the position of the animal along each axis. However, a plotter or PC data acquisition card is also needed. This system allows for standalone operation of up to seven units working simultaneously, and even more if a PC is used. Other previously reported systems, such as the Digiscan animal activity monitors (Omnitech, Columbus, $\mathrm{OH}$ ) used by Hunt, Zito, Erjavec, and Johnson (1994), not only performed motor activity data acquisition, but the current version also includes analysis software to calculate total distance traveled, number of horizontal movements, mean distance per movement, and so forth (Columbus Instruments, 2011).

Sharma (2003) reported about a computer-aided device that comprised "aktographs," opto-couplers, shift registers, and a Pentium PC to record the activity of insects and small mammals. In this device, the PC was dedicated to controlling the entire system for the duration of the experiment and was also used to analyze the collected data.

Other devices, including the MotorMonitor from Kinder Scientific, incorporate the hardware and software that are used to obtain metrics of movements or zones, and the results are reported in a spreadsheet-compatible file (Kinder Scientific, 2011). Still other authors, such as Wolfer, Madani, Valenti, and Lipp (2001), have developed custommade analysis software for the Windows (Microsoft Corp.) environment, to process data from a variety of commercially available tracking systems.

The use of the previously described systems for several simultaneous experiments leads to the need for a dedicated PC to control the activity boxes during the experiments, or at least to program the experimental conditions and to perform later data analysis. The only exception is the OptoVarimex-3 system, if fewer than eight units are used (Columbus Instruments, 2011).

Although video tracking systems have the advantage of gathering information that allows the study of complex behaviors (e.g., grooming, scratching, sniffing, smelling, and circling; Bonatz et al., 1987; Hopper et al., 1990; Noldus et al., 2001) and are versatile enough for measuring the behaviors of animals placed in enclosures of different shapes (circular or square open fields, Y-mazes, elevated plus mazes, water mazes, or complex environments with tunnels and partitions; Dielenberg et al., 2006; Noldus et al., 2001), they also suffer from some drawbacks. For example, their reliability greatly depends on the quality and quantity of lighting and on the contrast between the color of the animal's fur and the background (Bailoo et al., 2010; Dielenberg et al., 2006). Furthermore, the video tracking systems require a digital camera plus a computer with a hard disk with enough capacity to store the film. If the camera is analog, a digitizing interface would be needed (Bonatz et al., 1987). Another aspect to consider is light reflection caused by urine, which should be avoided (e.g., by attaching a black fabric at the bottom of the enclosure; Bonatz et al., 1987). Furthermore, since video systems detect "rearings" (animal standing upright on its two hind legs) on the basis of the area occupied by the rat body as seen from a vertical perspective, this may yield errors when a rat assumes other postures that produce this same effect, such as during grooming.

In contrast, photocell detection systems are immune to lighting or contrast problems but yield less precise path measurements, which may cause under- or overestimates of path length, according to the photocell spacing and animal size (Bailoo et al., 2010). In addition, since the emitting and receiving diodes must be precisely aligned for proper functioning, they must either be permanently fixed to the walls of the experimental setting or attached to rigid supports with a predefined geometric shape, which restricts their use to enclosures of the same shape and size.

A unidimensional array of LED/phototransistor pairs would be enough if the only intended measurement were the global amount of movement (Czech, 1984), without requiring information about the path length and trajectory. However, such systems do not discriminate between movements caused by locomotion and stereotypies (Clarke \& Smith, 1985).

In our approach, we considered creating a device equipped with a three-dimensional array of IRED/phototransistor pairs that did not require the use of a PC to program the experimental conditions or to control data acquisition during the experiment; for our purposes, a selfcontrolled device was a low-cost and versatile alternative. Even though PC costs have decreased, a more economical solution is to use a microcontroller, which only costs a few dollars. Furthermore, a microcontroller consumes less energy than an entire computer, is more reliable - since it has fewer components that can fail — and is immune to computer viruses. Additionally, an embedded control system does not require an operating system, which in PCs can give rise to 
both software and hardware compatibility problems when newer versions are used. Another issue is that PCs have much longer startup times. Thus, this prototype is governed by two reduced-instruction-set computer (RISC) microcontrollers. Experimental conditions can be programmed directly via a keyboard with only three buttons and a display, and the collected activity monitoring data are saved in ASCII format on a secure digital (SD) card.

In order to analyze the collected data, a computational system was developed using graphic programming (LabVIEW 8.0 National Instruments Corp.). This computational system is capable of creating a user profile analysis file that can be invoked to instruct the system to repeatedly perform the same group of analyses for animals in the same batch, avoiding the repetitive task of choosing the type of analysis, time intervals (in minutes), and file names for each rat. At the present time, the system includes seven programs: traveled distance $(\mathrm{cm})$, average movement speed $(\mathrm{cm} / \mathrm{s})$, time spent in vertical exploration (s), thigmotaxis (percentage of time traveled in periphery), time spent at each $x-y$ coordinate (min), time spent on vertical exploration at each $x-y$ coordinate (s), and frequency distribution of vertical exploration episodes of distinct durations. These last three analyses are made for the total duration of the experiment and constitute an innovative approach for motor behavior analysis.

\section{Materials and methods}

We developed a box with infrared sensors and associated circuits and firmware as a method for detecting changes in the motor activity of rats. The box itself (Fig. 1) is made of transparent acrylic (width, $50 \mathrm{~cm}$; length, $50 \mathrm{~cm}$; height, $50 \mathrm{~cm}$ ). A series of opto-couplers (infrared emitter and receiver pairs) are placed within black acrylic rails (light emitters on one of them and the corresponding detectors in

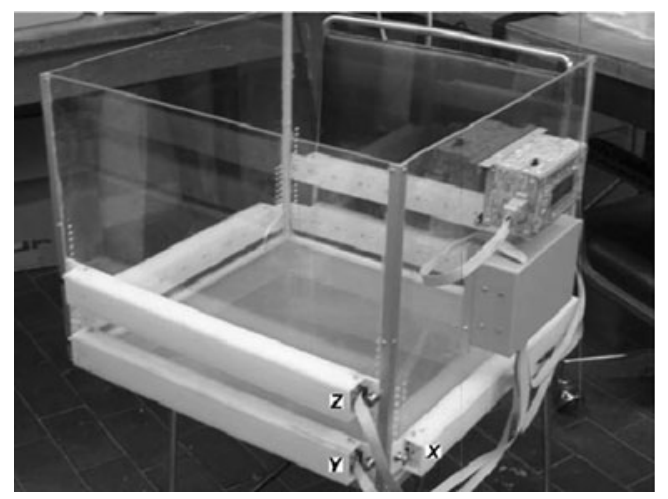

Fig. 1 Motor activity acrylic box. Three infrared device (either emitter or receiver) rails for each axis are shown. The corresponding pairs of device rails are mounted on the opposing walls another) which, in turn, are attached to the outside of the box on opposing walls. This permits establishing the location of an animal inside the box by means of Cartesian coordinates $(x, y)$. Nine opto-couplers are evenly spaced along each of the two plane axes. In addition, a third set of rails is used for the detection of "rearings" on a third axis $(z)$. An experimental time mark accompanies the set of coordinates that specify each movement in the box. The presence of the rat in the box floor is detected by the interruption of some of the coordinate-specific light beams. In fact, the animal will normally obstruct more than one beam in each of the $x$ - and $y$-axes, since the spacing between optocouplers is $5 \mathrm{~cm}$, and the rat body exceeds this length in many positions. Therefore, the system must select one $x$ and $y$-coordinate pair, and this is defined as the middle value in each case, or else the larger value when only two coordinates are obstructed. Besides, the emitter/receiver pairs (27 in all, 9 per axis) are mechanically and spectrally coupled in order to increase their efficiency.

The $x-y$ plane is thus divided into 81 possible coordinates (a $9 \times 9$ matrix array), which are sequentially scanned, along with the nine coordinates (a linear array) corresponding to the vertical ( $z$ ) axis ( $x, y, z=1$ to 9). That is, three detectors (one from each coordinate axis) are polled at the same time. The possible interruption of any of them is sensed individually.

Figure 2 shows a block diagram of the circuits that constitute the multiplexing and demultiplexing section. The consecutive selection of each detector (PT1302B/C2 phototransistor (Steren, México D.F.) and emitter (IR383 infrared emitting diode, Steren) pair on each axis is made with a multiplexerdemultiplexer circuit, consisting of a CD4051 single 8-channel multiplexer and a CD4066 quadruple analog bidirectional switch circuit (National Semiconductors Corp., Santa Clara, CA). Two such devices are required because of the number of opto-couplers (nine) to be controlled.

In order to avoid the noise problems associated with spurious light in the emitter-receiver link, as described by Clarke and Smith (1985), the infrared emission is frequency-modulated using the local oscillator of an LM567 phase-locked loop (PLL) tone decoder circuit (National Semiconductors Corp.). The infrared signal from the receiver passes through the demultiplexer circuit and is then

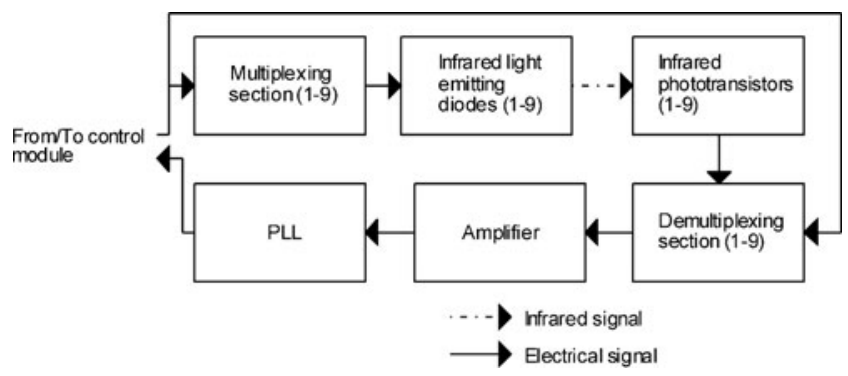

Fig. 2 Block diagram of the multiplexing-demultiplexing circuits 
amplified and sent to the PLL to be compared with its own internal frequency. A TTL (transistor-transistor logic) output from the PLL circuit produces a LOW logic level when a signal with this frequency is received, so that a phasesensitive closed loop is created. This circuit is reproduced three times, one for each coordinate axis.

The control module is constructed around a PIC16F877 RISC eight-bit microcontroller (Microchip Inc., Chandler, AZ). A 4-MHz system clock circuit was used.

This microcontroller is programmed to control the multiplexing-demultiplexing circuits that scan all of the $x-, y-, z$ coordinates (Fig. 3) from the rails of the activity box. The microcontroller sends a binary sequence (of a value from 0 to 8 ) to successively select each of the nine coordinates in each axis. It then waits for $30 \mathrm{~ms}$, to allow the PLL circuits to lock, and reads the binary outputs of each axis to establish which coordinates were obstructed. This reflects the instantaneous position of the animal, which is saved after having been confirmed. Validation consists of reading all the coordinates in the box three times. The purpose of this process is twofold: to establish the new position of the animal (middle value when three coordinates are obstructed or the largest one when only two coordinates are blocked) and, on the basis of this new position, to determine whether the movement is legitimate (i.e., if the rat changed its position by at least $5 \mathrm{~cm}$ or performed a rearing). The validated positions are saved every second in a 24LF256 electrically erasable programmable read-only memory (EEPROM; Microchip Inc.). The total memory storage capacity is $64 \mathrm{kB}$.

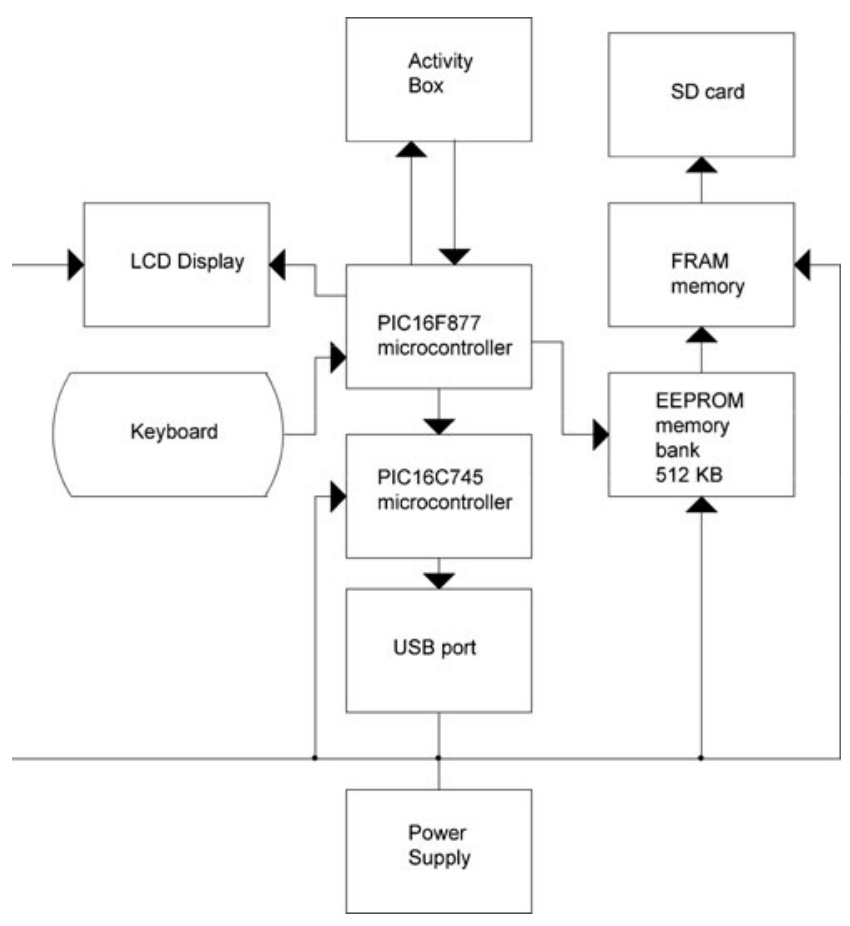

Fig. 3 Block diagram of the electronic control module
An AND 491 liquid crystal display (LCD) (Purdy Electronics Corp., Sunnyvale CA) and a set of three push-button switches (which constitute a keyboard) are also handled by the microcontroller. The display allows the user to see which coordinates are occluded by the rat, while at the same time it shows the elapsed experimentation time. The keyboard permits the user to introduce the experimentation time, to start or abort an experiment, or to save data onto an SD memory card. A ferroelectric random access memory (FRAM) FM24CL64 (Ramtron International Corp., Colorado Springs, CO) is used as a high-speed data buffer to transfer 512-byte blocks of data from the EEPROM to the SD card. This is necessary due to the time-access restrictions of this last device.

The second RISC microcontroller, type PIC16C745 (Microchip Inc.), receives serial data from the first microcontroller via its serial port, which are then transmitted via a universal serial bus (USB) port to a PC. This microcontroller performs the entire process needed to "enumerate" (identify and register) itself as a USB raw-data device.

All of the previously described circuits are powered by a $9-\mathrm{V}$ DC power supply source that is $5 \mathrm{~V}$ down-regulated. A 9.6-V/2.5-Ah NiMH rechargeable battery pack provides power during blackouts.

\section{Software}

Assembly language firmware for both microcontrollers was developed. One assembly program is used to instruct the PIC16F877 microcontroller to scan the coordinates of each axis, detect and validate the rat position, save the data in memory, show this experimental information on the LCD, keep track of the experimental time, and save the collected data as an ASCII (text) file on the SD memory card, for future transfer to a PC. A flow diagram of this program is shown in Fig. 4.

The interruption routine for the data acquisition of the rat movements begins by updating the coordinate number to be scanned. Then the status of the corresponding detector (obstruction or no obstruction) is obtained and compared with previous entries in order to discard invalid movements, such as repeated beam interruptions of the same set of coordinates. Finally, when a new valid movement is registered, the $x, y$, and $z$ values are saved in memory along with the time of occurrence.

The second assembly program instructs the PIC16C745 microcontroller (Fig. 3) to read serial data from the first microcontroller (PIC16F877), which can later be sent to a $\mathrm{PC}$ via a USB connection.

For the data analysis in a PC, a set of programs was developed in a graphical programming environment (LabVIEW). These programs are contained in a computational system that initially permits the creation of user profile analysis files. This type of file contains a selection of any 


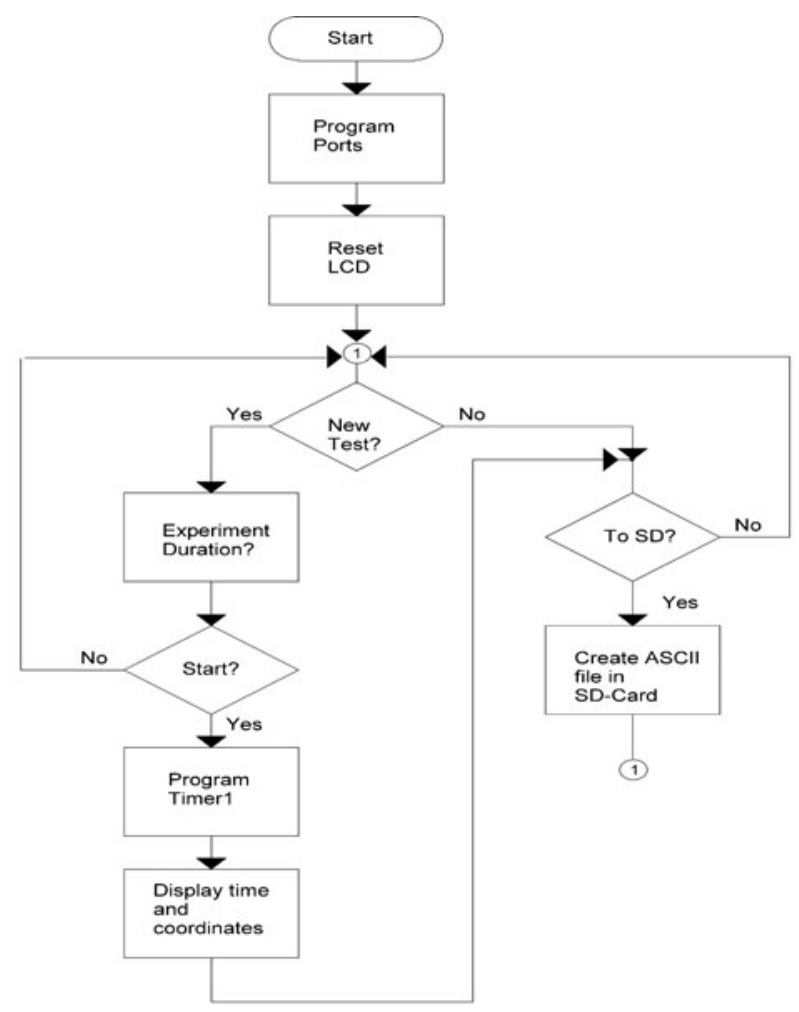

Fig. 4 Main software flow diagram

of the nine available analyses that the user wants to be executed. In addition, time intervals (in minutes) are selected, when applicable for the chosen analysis routines. A file name is defined for each selected analysis, and the system automatically adds a number ending for the file according to the time interval selected, in order to facilitate identification. The profile analysis file can be read each time the system is run to automatically reload all of the needed analyses, but it can also be modified or a new one created.

The system begins by creating a working directory in the root of the main hard disk of the $\mathrm{PC}$, and if it already exists, the system checks whether it is empty. If it is not, the user is prompted to empty the directory or to allow the system to delete every file in it. Next, the user is prompted to indicate the location of the SD file with the experimental data that were created during acquisition. The user is then asked to load an analysis profile, modify an existing one, or create a new one. Next, the system automatically creates a group of working files and performs each of the selected analyses. The files with the results are saved as text files in the same working directory, and they can be read by any editing or statistical software. After all of the analyses are finished, the system prompts the user to determine whether further data are to be analyzed. Otherwise, this ends the execution of the program.

(All of the software code related to this prototype is freely available from www.cirbiomedicas.uady.mx/content.php? op=labingbiomed).
System evaluation

An experimental assessment verified the performance of the developed system. This was also carried out to show the usefulness of the device in a pharmacological application. The experiments were videotaped and later analyzed by two independent, trained observers. The data from the electronic system were compared against the movements recorded by the observers.

The automated motor activity box was used to test a lowdose caffeine treatment with respect to motor and exploratory activity in six rats (File, Baldwin, Johnston, \& Wilks, 1988). Male Wistar rats (220-320 g) were maintained at a temperature of $23 \pm 1{ }^{\circ} \mathrm{C}$ under 12:12-h light:dark cycles (lights on at 07:00) for a week prior to the experiments. Food and water were available ad libitum. The recommendations of the Guide for the Care and Use of Laboratory Animals were strictly followed (National Research Council of USA, 1996).

Three rats were treated with vehicle (saline solution $0.9 \%$ ), and three others received caffeine at the dose of $10 \mathrm{mg} / \mathrm{kg}$. Both treatments were administrated by the i.p. route. After $30 \mathrm{~min}$ from the treatment application, spontaneous motor activity was recorded over $30 \mathrm{~min}$ using the activity box and a video camera mounted over it.

\section{Results}

The developed motor activity box (Fig. 1) was installed in our experimentation facilities (a room with light/dark timercontrolled cycles and temperature and ventilation regulation). The video camera was located on top of the box pointing downward, in order to obtain a complete view of the entire box floor, on which a $9 \times 9$ grid of lines interspaced by $5 \mathrm{~cm}$ was painted in order to facilitate the observational analysis.

All of the motor activity data were saved on an SD card in ASCII files, which are readable in any PC. An excerpt of such a file is shown in Table 1 (the top row-with time and

Table 1 A fragment of a data file as it is saved in the SD card

\begin{tabular}{llll}
\hline Time & $x$ & $y$ & $z$ \\
\hline 108 & 3 & 6 & 1 \\
109 & 3 & 7 & 0 \\
110 & 2 & 8 & 0 \\
111 & 3 & 9 & 0 \\
112 & 4 & 9 & 0 \\
114 & 5 & 9 & 0 \\
115 & 6 & 9 & 0 \\
116 & 7 & 9 & 0 \\
\hline
\end{tabular}


$x, y$, and $z$ as legends - is not part of the file, but is shown only for illustrative purposes).

The videotaped data in this work were first manually evaluated and then the recordings made by the electronic system were assessed using the developed software. Three motor activity analyses were performed: traveled distance, time spent in vertical exploration (rearing), and distance traveled in the periphery of the box floor (i.e., movements performed at coordinates 1 or 9 of either the $x$ - or $y$-axes, or both). Graphs were made using both the data recorded by the activity box and those reported by the two observers.

Figure 5 shows graphs corresponding to the first $10 \mathrm{~min}$ of activity in terms of the average traveled distance, which was calculated in each case according to the records obtained from the observers and from the developed system, and for both the vehicle- (A) and the caffeine- (B) treated rats, in 1-min intervals.

The results from both observers, as well as those from the developed system, show a high level of concordance in the reported values (Observer 1 vs. Observer 2, $r=.98, p<.0001$; Observer 1 vs. automated system, $r=.99 p<.0001$; Observer 2 vs. automated system, $r=.97, p<.0001$ ).

In Fig. 6, a comparison between the data from the automated system and the two observers regarding the vertical exploration time is presented for the vehicle- (A) and caffeine- (B) treated rats at 1-min intervals. Pairwise comparisons of the reports from the two observers as well as from the automated system yielded significant correlation values: Observer 1 versus Observer $2(r=.96, p<.0001)$; Observer 1 versus the developed system $(r=.87, p<.0018)$; Observer 2 versus the developed system $(r=.90, p<.0011)$.

Figure 7 illustrates the distance traveled by the rats in the box periphery (along the external borders of the box; i.e., at the 1 and $9 x$ - and/or $y$-coordinates). Since rats naturally seek sheltered places (in this case, the box borders), this measure serves as an index of anxiety. Both of the observers showed moderate concordance between them (Observer 1 vs. Observer 2, $r=.82, p<.0026$ ), as well as between Observer 1 and the developed system $(r=.83 p<.0007)$. The correlation between Observer 2 and the developed system was somewhat higher $(r=.97, p<.0001)$.

\section{Discussion}

The system offers several important features. For instance, the use of the PLL to generate the pulsed infrared link for the detection of the rat position renders recalibrations or adjustments unnecessary over time (the PLL locks on its own frequency, so any frequency drift will be present in the signal to be compared). Likewise, an advantage is the backup battery system, which allows experiments of up to $6 \mathrm{~h}$ to be completed without any data loss, should there be a power failure. Performing experiments while batteries are charging is also possible.

Using rats having weights within the specified range of the system $(220-320 \mathrm{~g})$ enabled the discrimination of real displacements from minor, local movements performed at the same positions, both in the $x-y$ plane and in the $z$ direction. The movement resolution of $5 \mathrm{~cm}$ (which is the spacing between opto-couplers) is sufficient for young adult rats.

The EEPROM memory holds the data of the last experiment even when the power is off. Therefore, they may be transferred to the SD card immediately after concluding an experiment, or at any time thereafter. The use of SD cards represents a convenient, compact, and economical means for data transfer.

All of the collected data are downloaded into an SD memory card in an ASCII text file. The card is formatted using the FAT16 file structure, which eases the data transfer to a PC, since this file format is recognized by the majority of current operating systems. In addition, the fact that the data are in a text file allows it to be opened by any statistical analysis program, in order to perform various behavioral analyses, such as traveled distance, velocity, and so forth.

As is shown in the results above, the system is capable of detecting and saving pharmacologically induced motor activity changes in rats. In Fig. 5 (traveled distance), the concordance between the results obtained by the observers and by the system is clear, in spite of the small differences in values due to the observation angle of the camera, which in some instances made it difficult to determine the real coordinate occupied by the rats. Furthermore, the differences in motor activity
Fig. 5 Graphs showing the average traveled distance $(M \pm$ $S E M)$, calculated with data obtained from the developed system and from the two independent, trained observers: (a) Rats treated with the vehicle only. (b) Rats treated with caffeine
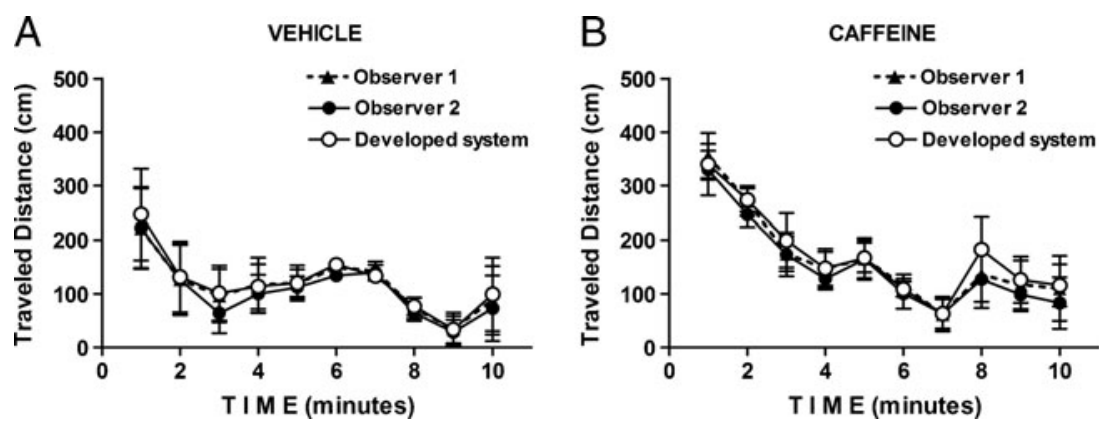
Fig. 6 Graphs showing the average exploration time ("rearings") $(M \pm S E M)$, calculated with data from both the developed system and the trained observers: (a) Rats treated with vehicle. (b) Rats treated with caffeine
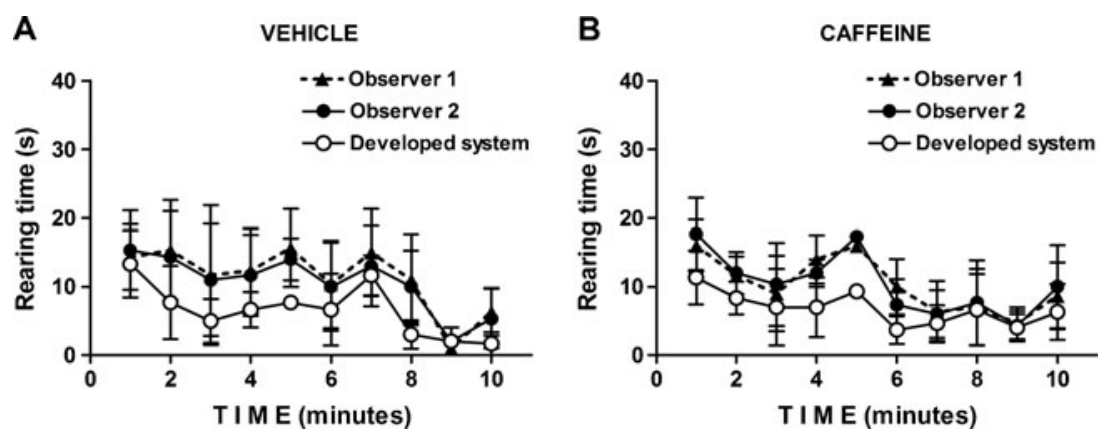

due to caffeine with respect to the vehicle group were clearly detected by the system. In this case, since the goal was to show the capability of the system of detecting and reporting the same information seen by the observers, only the first $10 \mathrm{~min}$ of the experiment are displayed.

However, some minor discrepancies between the distance measured by the observers and the developed system are evident at some time points. This is because the possibility of producing errors increases when the distance values are calculated manually, since this process involves repetitive mathematical operations and later data transcription.

Figure 6 shows the ability of the developed system to detect vertical activity during the initial $10 \mathrm{~min}$ in the box. The rearing times reported by the observers were on average larger than those recorded by the automatic system. A possible explanation for this is that they counted the time whenever the rat raised its forepaws above the floor, irrespective of the maximum height reached by the animal. In contrast, the electronic device only counted the time when the rat's body surpassed the fixed height of $15 \mathrm{~cm}$ from the floor where the $Z$ position detectors were situated. This would lead to an overestimation of the rearing time by the observers in comparison to the electronic system. Perhaps the use of a second camera facing the activity box laterally would have enabled the observers to record vertical movements more accurately. However, the use of a fixed minimum height to define a vertical movement is an advantage in terms of consistency for this type of measurement.
Figure 7 shows the distances traveled in the periphery of the box as reported by the observers and the developed system. Only the first $10 \mathrm{~min}$ are shown. As can be seen, the stimulant effect of caffeine leads to a slight decrease in the traveled distance. This is explained by the fact that under this condition, rats increase their movements in the central part of the box (the open area), which is in opposition to their natural behavior of seeking sheltered places (in this case, the box borders).

As with some of the most versatile commercial devices, this computational system allows for performing specific sets of analyses (according to the selected profile) for each group or groups of rats, thus saving time in this kind of repetitive task, particularly with large numbers of animals or when they are to be evaluated at different experimental stages.

In conclusion, this is a low-cost, standalone, easy-toduplicate system, useful for recording and analyzing ambulatory activity in rats, although it is not intended to capture fine motor activity (scratching, grooming of whiskers, face or forelegs, performing tight turns, etc.). For such stereotypic behaviors, a video-capture-andanalysis approach would be more appropriate. Another obvious limitation of the system is that it is not useful for social interaction studies (involving more than one animal within the experimental area at the same time). With respect to the maximum duration of the motor activity recording, the system has a 6-h limit. However, this electronic system is capable of faithfully recording movements and rearings, along with the time marks indicating when each event occurred. Furthermore, its
Fig. 7 Graphs showing the average distance traveled in the periphery of the developed system $(M \pm S E M)$, calculated with data from both the activity box and the two trained observers. (a) Rats treated with vehicle. (b) Rats treated with caffeine
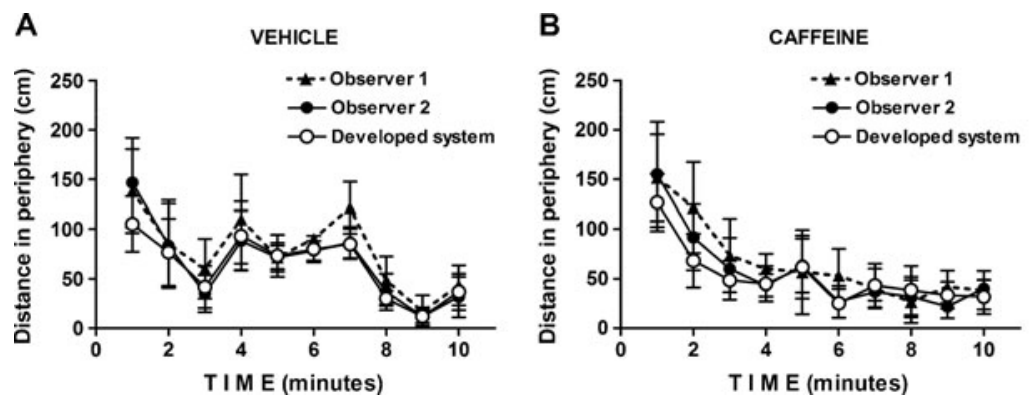
reduced construction cost (common hardware and electronic devices) makes it easy and affordable to reproduce, enabling the execution of several simultaneous experiments. A single system can be constructed for around US $\$ 80$, including all of the necessary hardware components. As can be seen in the results, the developed system is able to detect and report changes in motor activity, with accuracy comparable to that of trained observers. Furthermore, the automated analysis system can extract user-specified information serially for large numbers of animals, thus saving time. In addition, the system avoids introducing behavioral changes in the animal related to the presence of the researcher during the experiment. Moreover, as with other automated systems, motor activity experiments can be recorded in this way with less variability than by direct observation, and errors due to fatigue or lack of concentration can be eliminated.

Author note The authors declare that they have no competing financial interests.

\section{References}

Bailoo, J. D., Bohlen, M. O., \& Wahlsten, D. (2010). The precision of video and photocell tracking systems and the elimination of tracking errors with infrared backlighting. Journal of Neuroscience Methods, 188, 45-52.

Bonatz, A., Steiner, H., \& Huston, J. P. (1987). Video image analysis of behavior by microcomputer: Categorization of turning and locomotion after 6-OHDA injection into the substantia nigra. Journal of Neuroscience Methods, 22, 13-26.

Brockwell, N. T., Ferguson, D. S., \& Beninger, R. J. (1996). A computerized system for the simultaneous monitoring of place conditioning and locomotor activity in rats. Journal of Neuroscience Methods, 64, 227-232.

Clarke, R. L., \& Smith, R. F. (1985). An infrared device for detecting locomotor activity. Behavior Research Methods, Instruments, \& Computers, 17, 519-525.

Columbus Instruments. (2011). Animal activity meter: Opto-varimex-3. Retrieved September 29, 2011, from www.colinst.com/brief.php? id $=2$

Czech, D. A. (1984). A versatile integrated circuit activity monitor for small animals. Physiology and Behavior, 32, 871-874.

Dielenberg, R. A., Halasz, P., \& Day, T. A. (2006). A method for tracking rats in a complex and completely dark environment using computerized video analysis. Journal of Neuroscience Methods, 158, 279-286.

File, S. E., Baldwin, H. A., Johnston, A. L., \& Wilks, L. J. (1988). Behavioral effects of acute and chronic administration of caffeine in the rat. Pharmacology Biochemistry and Behavior, 30, 809-815.

Gapenne, O., Simon, P., \& Lannou, J. (1990). A simple method for recording the path of a rat in an open field. Behavior Research Methods, Instruments, \& Computers, 22, 443-448.

Geyer, M. A., Russo, P. V., \& Masten, V. L. (1986). Multivariate assessment of locomotor behavior: Pharmacological and behavioral analyses. Pharmacology Biochemistry and Behavior, 25, $277-288$

Golani, I., Benjamini, Y., \& Eilam, D. (1993). Stopping behavior: Constraints on exploration in rats (Rattus norvegicus). Behavioural Brain Research, 53, 21-33.

Hopper, D. L., Kernan, W. J., \& Wright, J. R. (1990). Computer pattern recognition: An automated method for evaluating motor activity and testing for neurotoxicity. Neurotoxicology and Teratology, 12, 419-428.

Hunt, J. R., Zito, C. A., Erjavec, J., \& Johnson, L. K. (1994). Severe or marginal iron deficiency affects spontaneous physical activity in rats. American Journal of Clinical Nutrition, 59, 413-418.

Kinder Scientific. (2011). MotorMonitor. Retrieved November 30, 2011, from www.kinderscientific.com/motor_host_software.html

Kramer, M. K. (1998). Inexpensive motion detectors for quantification of animal activity. BioTechniques, 25, 385-388.

Masuo, Y., Matsumoto, Y., Morita, S., \& Noguchi, J. (1997). A novel method for counting spontaneous motor activity in the rat. Brain Research Protocols, 1, 321-326.

National Research Council of USA. (1996). Guide for the care and use of laboratory animals. Washington, DC: National Academy Press.

Noldus, L. P. J. J., Spink, A. J., \& Tegelenbosch, R. A. J. (2001). EthoVision: A versatile video tracking system for automation of behavioral experiments. Behavior Research Methods, Instruments, \& Computers, 33, 398-414. doi:10.3758/BF03195394

Pan, W. H., Lee, C. R., \& Lim, L. H. (1996). A new video path analyzer to monitor travel distance, rearing, and stereotypic movement of rats. Journal of Neuroscience Methods, 70, 39-43.

Sams-Dodd, F. (1995). Automation of the social interaction test by a video-tracking system: Behavioural effects of repeated phencyclidine treatment. Journal of Neuroscience Methods, 59, 157-167.

Schwarting, R. K. W., Goldenberg, R., Steiner, H., Fornaguera, J., \& Huston, J. P. (1993). A video image analyzing system for openfield behavior in the rat focusing on behavioral asymmetries. Journal of Neuroscience Methods, 49, 199-210.

Sharma, V. K. (2003). A simple computer-aided device for monitoring activity of small mammals and insects. Biological Rhythm Research, 33, 3-12.

Vittorio, P., Scannapieco, E., \& Renzi, P. (2006). Validation of a microwave radar system for the monitoring of locomotor activity in mice. Journal of Circadian Rhythms, 4, 1-8.

Wolfer, D. P., Madani, R., Valenti, P., \& Lipp, H. P. (2001). Extended analysis of path data from mutant mice using the public domain software Wintrack. Physiology and Behavior, 73, 745-753. 